\title{
Study of Lipocalin-2 Associated with Neutrophilic Gelatinases (UNGAL) in the Urine in Children with the Microbial Inflammatory Diseases of Kidneys and Urinary Tract
}

\author{
E. D. Bondarenko', A. V. Eremeeva², V. V. Dlin ${ }^{3}$, A. A. Korsunsky4, S. B. Orekhova5, \\ S. Gurbanova ${ }^{6}$ \\ ${ }^{1}$ Postgraduate Student of the Chair of Pediatrics and Children's Infectious Diseases, SBEI HPE I.M. Sechenov \\ First MSMU, Flat 7, 18/3 Malye Kamenshiki St., Moscow \\ ${ }^{2}$ Docent of the Chair of Pediatrics and Children's Infectious Diseases, SBEI HPE I.M. Sechenov First MSMU, \\ 8/2 Trubetskaya St., Moscow \\ ${ }^{3}$ Deputy Director for Science, Scientific Research Clinical Institute of Pediatrics, N.I. Pirogov Russian National \\ Research Medical University, RNRMU, 2 Taldomskaya St., Moscow \\ ${ }^{4}$ Head of Pediatrics and Children's Infectious Diseases Department, SBEI HPE I.M. Sechenov First MSMU, 8/2 \\ Trubetskaya St., Moscow \\ ${ }^{5}$ Pediatric Department, G.N. Speransky City Children's Hospital № 9, 29 Shmitovskiy Passage, Moscow \\ ${ }^{6}$ Medical Plaza Clinic, 75A Vagifa Av., Baku, Azerbaijan \\ Email: alenkabond@gmail.com, alinaeremeeva@yandex.ru,vvdlin@mail.ru,dr_kaa@mail.ru, \\ dgkb9nefrolog@yandex.ru, info@medplaza.az
}

Received 20 October 2015; accepted 11 December 2015; published 18 December 2015

\begin{abstract}
Purpose of the study: Research of the clinical and diagnostic significance of determination of Lipocalin-2 associated with neutrophilic gelatinases (UNGAL) in the urine of children with urinary tract infection (UTI) and pyelonephritis. Materials and methods: We examined 30 children with acute pyelonephritis and UTI aged 1 to 16 years (average age $7.32 \pm 4.52$ ) including 26 girls and 4 boys. Verification of the diagnosis was conducted on the basis of clinical and laboratory data, medical history and instrumental examination of patients. All children were divided into 2 groups: $1^{\text {st }}$ group-15 children with acute pyelonephritis, $2^{\text {nd }}$ group-15 children with urinary tract infection. UNGAL was measured in the urine by enzyme-linked immunosorbent assay (EISA) (BioVendor Laboratoty Medicine). Results: It is found, that the urine level of NGAL depends on the damage degree of renal parenchyma. The correlation of medium strength was found between the excretion level of uNGAL during the acute period of pyelonephritis and the detection of renal scars according to the DMSA-nephroscintigraphy data. In the group of children with the acute pyelonephritis the direct correlation of medium strength was found between the excretion level of uNGAL/creatinine and leukocytosis value and also with the CRP blood level. Conclusion: The results


allow us to recommend the determination of the excretion level of uNGAL/creatinine as an additional non-invasive marker for the early detection of renal parenchyma injury.

Keywords

\author{
Lipocalin-2, Associated with Neutrophilic Gelatinases (NGAL), Acute Pyelonephritis, Urinary Tract \\ Infection, Children
}

\title{
1. Introduction
}

In the recent years there has been an increase in the frequency of urinary system diseases in children, whose share in the structure of microbial inflammatory diseases of kidneys and urinary tract is up to $80 \%$.

The prevalence of infectious and inflammatory diseases of the urinary system in childhood in the Russian Federation is 18 cases per 1000 pediatric population [1] [2]. The debut of this group of diseases is often observed on the background of upper respiratory tract infections RTI, intestinal infections , vaccination or exposure.

Urinary tract infections (UTI) are among the most common infectious diseases faced by pediatricians [3-[5]. Early diagnostics and treatment of UTI are very important [6] [7].

The problem of improving the diagnosis of the UTI and pyelonephritis in children continues to be one of the most urgent in the pediatric nephrology.

Because of non-specific clinical symptoms the laboratory and instrumental methods of research have great importance in the diagnosis of inflammatory diseases of the urinary tract.

Minimally invasive method with high sensitivity should be preferred in the examination of children with suspected urinary tract infection. The development and the search for the new biomarkers in urine for verification of bacterial inflammation in the kidney, which in the future may lead to the development tubulointerstitial fibrosis, is an important task in pediatric nephrology.

New potential biomarkers of bacterial inflammation have been recently proposed, including interleukins, enzymes, prostaglandins, Lipocalin-2, associated with neutrophilic gelatinases (NGAL), kidney injury molecules (KIM-1) and some others.

Among the most interesting and less studied is Lipocalin-2, associated with neutrophilic gelatinases (NGAL) or siderocalin, which was first identified as a component of specific neutrophilic granules. NGAL goes into the blood plasma from secondary granules of the activated neutrophils, but it can be synthesized in different organs and different cell types. NGAL is a protein component of an acute phase of inflammatory response, associated with the innate immune responses, differentiation, neogenesis and survival of cells [9] [10]. It is found that the plasma NGAL is freely filtered by glomerulus, but then largely reabsorbed in the proximal tube by endocytosis. For this reason NGAL "renal" pool synthesized in kidneys prevails in urine, this pool of NGAL doesn't flow into the blood circulation and is excreted completely into the urine. It is shown that in case of proximal tubules injury the fast increase of the mRNA, encoding urine NGAL (uNGAL), happens in an upward knee of Henle loop and collecting ducts. Its main functions are bacterial infections resistance and stimulation of damaged cells proliferation, first of all, epithelial [8] [11] [12].

There are reports about urine NGAL level (uNGAL) in children with kidney diseases in literature. According to the researchers' data at the uNGAL level of $20 \mathrm{ng} / \mathrm{ml}$ urinary tract infection was diagnosed with $97 \%$ sensitivity and $76 \%$ specifity. Authors believe that uNGAL can be used as a new sensitive marker for the early verification of urinary tract infection [13]. In another study, where a comparison between the uNGAL level, urine culture and the UTI symptoms was being made, the uNGAL urine level of $5.75 \mathrm{ng} / \mathrm{ml}$ was taken as diagnostically significant. No statistically significant difference $(p=0.064)$ between the uNGAL levels in children with the confirmed and non-confirmed UTI was received [14]. Analysing the results of this study it should be noted that an increase of uNGAL excretion level was associated only with symptoms of the UTI and a positive urine culture, while signs of kidney tubulointersticial department injury were not counted. According to other authors uNGAL excretion increase in microbial inflammatory changes in proximal tubules is lower than the uNGAL levels caused by ischemia-reperfusion of kidney parenchyma, typical for acute kidney injury (AKI), acute tubular necrosis or acute 
tubulointersticial nephritis [15]. Possibly the lack of information regarding urine concentration of this marker in different kidney injuries leads to contradictory data on the diagnostic value of uNGAL excretion level.

The purpose of our study was the research of the clinical and diagnostic significance of determination of Lipocalin-2 associated with neutrophilic gelatinases (uNGAL) in the urine of children with the urinary tract infection and pyelonephritis.

\section{Materials and Methods}

In the nephrology department of G.N. Speransky City Children's Hospital MHD 30 children with inflammatory diseases of urinary system organs aged 1 to 16 years (average age $7.32 \pm 4.52$ ) including 26 girls and 4 boys were examined.

All patients were examined using clinical, instrumental and laboratory methods. Along with the standard laboratory methods (clinical blood and urine tests, urine culture, urea and creatinine determination in blood, total blood protein, urine creatinine, Zimnitsky test for kidneys' concentrating ability) special methods such as the urine level of Lipocalin-2 associated with neutrophilic gelatinases (UNGAL) were used. NGAL level was measured in the morning urine by enzyme-linked immunosorbent assay (ELISA) (BioVendor Laboratory Medicine) according to the manufacturer's instructions. The study was conducted on an automated microplate reader. NGAL urine levels expressed in nanograms per milliliter, detection limit $<0.1 \mathrm{ng} / \mathrm{ml}$. For the standartisation of NGAL urine level we determined creatinine level in each urine sample and NGAL urine level was converted to creatinine mg. Instrumental study included ultrasonography of kidneys and urine bladder. Voiding cystourethrography was performed in children who had acute pyelonephritis 3 and more months before it. Static scintigraphy was perfomed at least 6 months (from 6 to 9 months) after pyelonephritis attack or the UTI by using radionuclide Technetium-99m-Dimercaptosuccinic Acid (DMSA) with activity of 100 - 150 MBk depending on child's age and weight (Federal State Budgetary Institution Russian Scientific Center of Roentgenoradiology (RSCRR) of the Ministry of Healthcare of the Russian Federation).

Statistical analysis of the results was performed with the program Statistica for Windows 6.0 with the expectation of contingency tables with the definition of qualitative characteristics $\chi^{2}$ and correlation analysis. Differences were considered reliable indicators at $p<0.05$.

\section{Results}

As a result of comprehensive clinical laboratory and instrumental examination 2 groups were allocated. The first group included children with acute pyelonephritis $(n=15)$, the second group consisted of children with urinary tract infection $(n=15)$. Average age of the first group was $8.5 \pm 0.6$ years, of the second group $-9.3 \pm 0.9$ years. Girls prevailed in both groups (Table 1).

All children with acute pyelonephritis marked symptoms of intoxication, fever from subfebrile to febrile values. 53.3\% of patients during hospitalisation complained only about fever, making it difficult to diagnose and determing late hospitalization. 7 patients (46.6\%) has abdominal pain syndrome, manifestating as anxiety in young children, some of the children's condition required surgical consultation. 3 (20\%) patients with pyelonephritis had disuria. In the group of children with urinary tract infection intoxication symptoms were less expressed, fever usually was subfebrile, 14 (93.3\%) patients marked disuria in their clinical picture.

Laboratory tests of the first group revealed massive (more than 100 in sight) leukocyturia in 13 (86.7\%) children, moderate (less than 100 in sight) leukocyturia-in 2 (13.3\%) children. Microbiological examination of urine showed sowing of gram-negative flora (Escherichia coli, Klebsiella, Enterobacter) in 6 (40\%) children.

$12(80 \%)$ children of the second group had moderate leukocyturia in urine analyses, massive leukocyturia was detected only in 3 patients (20\%). 5 (33.4\%) children had urine sowing of gram-negative flora (Escherichia coli).

Tubular disorders prevailed in children with pyelonephritis and were characterised by hyposthenuria, isosthenuria and nocturia. In the group of children with the UTI the decrease of concentrating renal function was registered much less often (Table 2).

Analyzing leukocytes blood level, ESR acceleration, CRP increase and leukocyturia it should be noted that children with active current microbial inflammatory process in the renal tissue had higher rates than children from the UTI group (Table 3, Image 1). Violation of the concentrational function is found in the children with hyperoxaluria or hypercalciuria. 


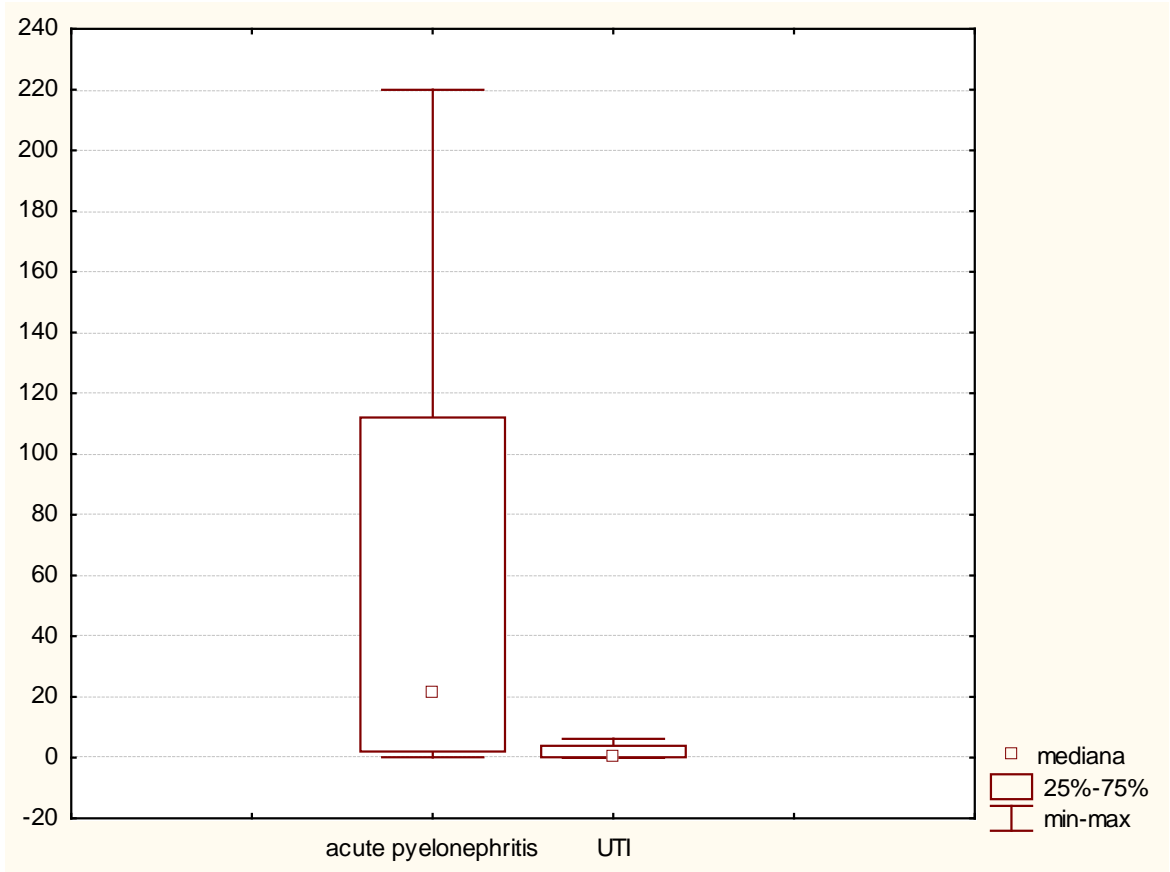

Image 1. The level of CRP in children with acute pyelonephritis and urinary tract infection on the day of admission to the hospital.

Table 1. Distribution of children by age and sex.

\begin{tabular}{ccccc}
\hline \multirow{2}{*}{ Groups of patients } & \multicolumn{2}{c}{ First group $\mathrm{n}=15$} & \multicolumn{2}{c}{ Second group $\mathrm{n}=15$} \\
\cline { 2 - 5 } & $\mathrm{n}$ & $\%$ & $\mathrm{n}$ & 6.66 \\
\hline $1-2$ years & 2 & 13.33 & 7 & 46.67 \\
$3-7$ years & 8 & 53.33 & 7 & 46.67 \\
$8-15$ years & 5 & 33.44 & & 20.00 \\
Sex & & & 3 & 80.00 \\
Boys & 1 & 6.66 & 12 & \\
Girls & 14 & 93.44 & & \\
\hline
\end{tabular}

Table 2. Characteristics of kidneys’ functional state.

\begin{tabular}{ccccc}
\hline \multirow{2}{*}{ Groups of patients } & \multicolumn{2}{c}{ First group $\mathrm{n}=15$} & \multicolumn{2}{c}{ Second group $\mathrm{n}=15$} \\
\cline { 2 - 5 } & $\mathrm{n}$ & $\%$ & $\mathrm{n}$ & 6.6 \\
\hline Nocturia & 3 & 20.0 & 1 & 13.3 \\
Hyposthenuria & 10 & 66.7 & 2 & 6.6 \\
Isosthenuria & 2 & 13.3 & 1 & 26.6 \\
In total & 15 & 100 & 4 & \\
\hline
\end{tabular}

Table 3. Laboratory findings in children with acute pyelonephritis and the UTI during admission.

\begin{tabular}{|c|c|c|c|}
\hline Options & First group & Second group & $p$ \\
\hline Blood leukocytes & $14.7 \pm 1.32$ & $8.3 \pm 0.98$ & $p<0.05$ \\
\hline Erithrocyte sedimentation rate & $36 \pm 5$ & $15 \pm 2$ & $p<0.05$ \\
\hline C-reactive protein & $60 \pm 21$ & $1.9 \pm 0.7$ & $p<0.05$ \\
\hline Leukocyturia (in sight) & $234 \pm 98$ & $35 \pm 17$ & $p<0.05$ \\
\hline
\end{tabular}


The uNGAL level in children with active current microbial inflammatory process in kidney tissue was significantly higher (Image 2) than in children from the UTI group $(19.73 \pm 12.37$ and $0.85 \pm 0.54 \mathrm{ng} / \mathrm{mg}$ of creatinine, respectively, $p<0.05$ ). The uNGAL/ Cr excretion level in children with the UTI did not exceed $2.5 \mathrm{ng} / \mathrm{ml}$ and in patients with pyelonephritis the uNGAL/Cr excretion was $5.7 \mathrm{ng} / \mathrm{ml}$ and more.

In the group of children with acute pyelonephritis a direct correlation of moderate strength $(\mathrm{r}=0.72 ; p<0.05)$ was between the uNGAL/Cr excretion level and the amount of blood leukocytes, as well as the CRP blood level ( $r$ $=0.67 ; p<0.05$ ).

To assess kidneys' function we defined glomerular filtration rate by Schwartz in children of the two groups (Table 4). All children with the UTI and most of the children (80\%) with acute pyelonephritis had normal levels of GFR, however 2 (13.3\%) children were diagnosed with hyperfiltration and 1 (6.7\%) child was diagnosed with moderate decrease of glomerular filtration rate. In the first group of children a weak correlation between GFR and the uNGAL/Cr was found $(\mathrm{r}=0.46 ; p<0.05)$.

All children of the first group had voiding cystourethrography 3 months after the decrease in pyelonephritis activity, according to its results no vesicoureteral refluxes and malformations of urethra and urinary bladder were found. 13 (86.6\%) children with pyelonephritis 6 and more months after its attack had sclerotic foci in kidneys' parenchyma found by static nephroscintigraphy. 7 of them had 1 - 2 foci, 4 patients had 2 - 3 foci, 2 children were diagnosed with 4 foci without decrease of accumulative-excretory function of kidneys. In the group of children with the UTI sclerotic foci and accumulative-excretory kidneys' disfunctions were not found by using static nephroscintigraphy. Strong positive correlation $(\mathrm{r}=0.71 ; p<0.05)$ was established between the

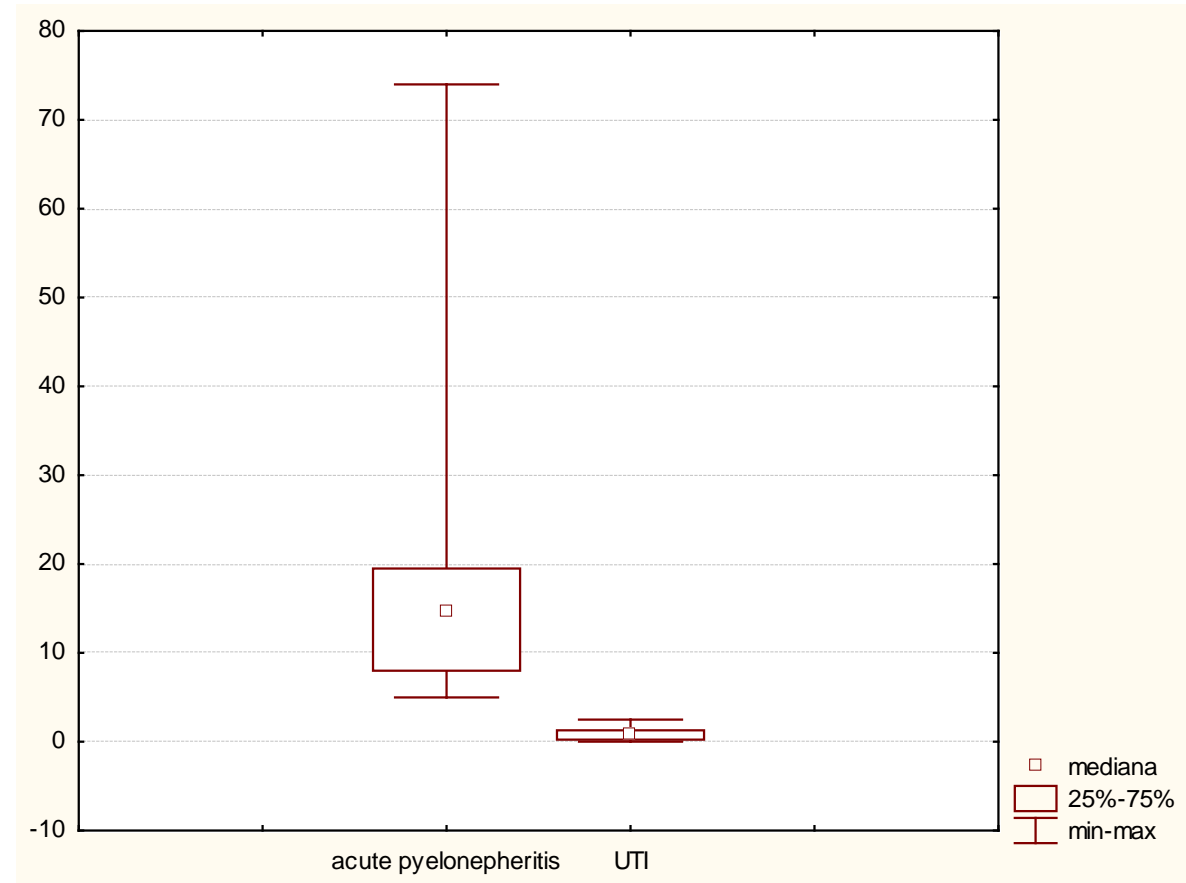

Image 2. The level of uNGAL/Cr in children with acute pyelonephritis and urinary tract infection at admission to the hospital.

Table 4. Indicators of Glomerular Filtration rate (Schwartz formula).

\begin{tabular}{ccccc}
\hline \multirow{2}{*}{ GFR rate $\mathrm{ml} / \mathrm{min}$} & \multicolumn{2}{c}{ Acute pyelonephritis $(\mathbf{n}=\mathbf{1 5})$} & UTI $(\mathbf{n}=\mathbf{1 5})$ \\
\cline { 2 - 5 } & Abs.n. & $\mathbf{\%}$ & Abs.n. & \% \\
\hline $121-140$ & 2 & 13.3 & 0 & 0 \\
$90-120$ & 12 & 80.0 & 15 & 100 \\
Менее 90 & 1 & 6.7 & 0 & 0 \\
\hline
\end{tabular}


uNGAL increase level during the acute pyelonephritis and damage degree of renal parenchyma in children with acute pyelonephritis according to the results of the static nephroscintigraphy.

\section{Discussion}

Early diagnosis of the presence and degree of renal parenchyma damage, especially in the young children, is very difficult, but the correct assessment allows us to choose an adequate therapy. Published data about the possibility of non-invasive assessment methods vary. Inflammation that occurs on the background of the tissue damage is accompanied with a production of inflammatory cytokines, acute phase proteins, the excretion of enzymes, the identification of which may indicate severity of the inflammation, increases at the destruction of the tubules [16]-[18]. In our study we found that the uNGAL urine level depends on the degree of renal parenchyma damage. Positive correlation was revealed between the uNGAL/Cr and such acute phase indicators in blood as CRP and leukocytosis in children with acute pyelonephritis. In the patients of the first group symptoms of intoxication prevailed in clinical picture, febrile fever was more common, erythrocyte sedimentation rate significantly accelerated, tubular renal dysfunctions and massive leukocyturia were diagnosed.

Static DMSA-nephroscintigraphy is the "gold" standard of renal parenchyma damage diagnostics and of determination of the amount and localisation of nephrosclerosis foci [19] [20]. However, despite being highly informative method of renal parenchyma damage diagnostics, nephroscintigraphy currently remains an expensive method of examination and the possibility of its implementation is limited even in foreign clinics and diagnostic centres [21].

The dependence between the uNGAL/Cr excretion level and the amount of renal scars in children after acute pyelonephritis established according to the clinical laboratory data during an acute period of disease, confirmed by the static DMSA-nephroscintigraphy. To form a cohort of patients in need of static DMSA-nephroscintigraphy after acute pyelonephritis we can use high the uNGAL/Cr excretion level as a marker at the acute phase of the disease. Category of patients undergone severe course of acute pyelonephritis also needs non-invasive method of monitoring the state of tubular nephron part, therefore more research of the uNGAL excretion dynamics is needed in the large patients' groups.

Received data points to the need of further research of the uNGAL significance as one of the diagnostic markers of renal parenchyma damage. Considering this it is important to determine the diagnostic significance of the uNGAL at the exacerbation of chronic microbial and inflammatory diseases of the urinary system, latent inflammatory process, at the development of pyelonephritis on the background of the congenital abnormalities of the urinary system, as well as an early marker of renal tissue scarring.

\section{Conclusion}

The determination of the uNGAL excretion in urine is available and non-invasive method for the early detection of renal parenchyma damage, an increase of differential diagnosis quality and a choice of curative tactics of the patients with microbial inflammatory diseases of the urinary system. However, further studies in the large groups of patients, conducted in dynamics, are required to confirm the results.

\section{References}

[1] Al-Ismaili, Z., Palijan, A. and Zappitelli, M. (2011) Biomarkers of Acute Kidney Injury in Children: Discovery, Evaluation, and Clinical Application. Pediatric Nephrology, 26, e29-e40.

[2] Biggi, A., Dardanelli, L., Pomero, G., et al. (2011) Acute Renal Cortical Scintigraphy in Children with a First Urinary Tract Infection. Pediatr Nephrol, 16, e733-e738.

[3] Devarajan, P. (2008) Neutrophil Gelatinase-Associated Lipocalin-An Emerging Troponin for Kidney Injury. Nephrology Dialysis Transplantation, 23, e3737-e3743.

[4] Gurgoze, M.K., Akarsu, S., Yilmaz, E., et al. (2005) Pro Inflammatory Cytokines and Pro Calcitonin in Children with Acute Pyelonephritis. Pediatr Nephrol, 20, e1445-e1448.

[5] Ignatova, M.S. (2004) Pathology of the Urinary System in Children (Modern Aspects). Nefrologija i dializ, 6, $127-132$.

[6] Jerman, M.V. (2010) Nephrology Childhood. SPb: SpecLit, 683.

[7] Karavanaki, K., Haliotis, F.A. and Haliotis, F.A. (2007) DMSA Scintigraphy in Febrile Urinary Tract Infections Could Be Omitted in Children with Low Procalcitonin Levels. Infectious Diseases in Clinical Practice, 15, e377-e381. 
[8] Kim, B.H., Yu, N., Kim, H.R., et al. (2014) Evaluation of the Optimal Neutrophil Gelatinase-Associated Lipocalin Value as a Screening Biomarker for Urinary Tract Infections in Children. Ann Lab Med., 34, e354-e359.

[9] Kjeldsen, L., Bainton, D.F., Sengelov, H., et al. (1993) Structural and Functional Heterogeneity among PeroxidaseNegative Granules in Human Neutrophils: Identification of a Distinct Gelatinase-Containing Granule Subset by Combined Immunocytochemistry and Subcellular Fractionation. Blood, 82, e3183-e3191.

[10] Kjeldsen, L., Johnsen, A.H., Sengelov, H., et al. (1993) Isolation and Primary Structure of NGAL, a Novel Protein Associated with Human Neutrophil Gelatinase. J. Biol. Chem., 268, e10425-e10432.

[11] Korovina, N.A. (2007) Pyelonephritis. Diagnosis and Treatment of Nephropathy in Children. Editors Ignatova M.S. end Korovina N. A. M.: Gjeotar-Media, 164-199.

[12] Lucas-Sáez, E., Ferrando-Monleón, S., Marín-Serra, J., et al. (2014) Predictive Factors for Kidney Damage in Febrile Urinary Tract Infection. Usefulness of Procalcitonin. Nefrologia, 34, e451-e457.

[13] Mishra, J., Ma, Q., Prada, A., et al. (2003) Identification of Neutrophil Gelatinase-Associated Lipocalin as a Novel Early Urinary Biomarker for Ischemic Renal Injury. Clin. J. Am. Soc. Nephrol, 14, e2534-e2543.

[14] Mitsnefes, M., Kathman, T., Mishra, J., et al. (2007) Serum NGAL as a Marker of Renal Function in Children with Chronic Kidney Disease. Pediatr Nephrol, 22, e101-e108.

[15] Mori, K., Lee, H.T., Rapoport, D., et al. (2005) Endocytic Delivery of Lipocalinsiderophore-Iron Complex Rescues the Kidney from Ischemia-Ischemia-Reperfusion Injury. J Clin Invest, 115, e610-e621.

[16] Papajan, A.V., Anichkova, I.V., Kosheleva, L.N. and Savenkova, N.D. (2008) Microbial Inflammatory Diseases of the Urinary Organs. Clinical Pediatric Nephrology Age. Editors Papajan A.V. end Savenkova N.D. SPb.: Levsha, 396-419.

[17] Piepsz, A., Colarinha, P., Gordon, I., et al. (2001) Peadiatric Committee of the European Association of Nuclear Medicine. Guidelines for 99-mTc-DMSA Scintigraphy in Children. Eur J Nucl Med, 28, e37-e41.

[18] Pugachev, A.G. (2009) Pediatric Urology. M.: GJeOTAR-Media, 333.

[19] Roberts, K.B. (2011) Urinary Tract Infection: Clinical Practice Guideline for the Diagnosis and Management of Initial UTIs in Febrile Infants and Children Aged 2 to 24 Months. Pediatrics, 128, e595-e610.

[20] Vjalkova, A.A., Gricenko, V.A. and Gordienko, L.M. (2010) Urinary Tract Infection in Children: New Solutions to an Old Problem. Nefrologija, 4, 63-75.

[21] Yilmaz, A., Sevketoglu, E., Gedikbasi, A., et al. (2009) Early Prediction of Urinary Tract Infection with Urinary Neutrophil Gelatinase Associated Lipocalin. Pediatr Nephrol., 24, e2387-e2392. 